\title{
High-frequency Cross-correlation in a Set of Stocks
}

\author{
Giovanni Bonanno ${ }^{1}$, Fabrizio Lillo ${ }^{1}$ and Rosario N. Mantegna ${ }^{1,2}$ \\ ${ }^{1}$ Istituto Nazionale per la Fisica della Materia, Unità di Palermo, Facoltà di Ingegneria, Università di Palermo, \\ Viale delle Scienze, I-90128 Palermo, Italy \\ and \\ ${ }^{2}$ Dipartimento di Fisica e Tecnologie Relative, Università di Palermo, Viale delle Scienze, I-90128 Palermo, Italy
}

\begin{abstract}
The high-frequency cross-correlation existing between pairs of stocks traded in a financial market are investigated in a set of 100 stocks traded in US equity markets. A hierarchical organization of the investigated stocks is obtained by determining a metric distance between stocks and by investigating the properties of the subdominant ultrametric associated with it. A clear modification of the hierarchical organization of the set of stocks investigated is detected when the time horizon used to determine stock returns is changed. The hierarchical location of stocks of the energy sector is investigated as a function of the time horizon.
\end{abstract}

\section{INTRODUCTION}

The presence of a high degree of cross-correlation between the synchronous time evolution of a set of stocks is a well known empirical fact observed in financial markets [1] 3]. The typical time horizon used to determine the cross-covariance or the correlation coefficient of a pair of time series of the price of a financial asset is one trading day. For this time horizon correlation coefficients as high as 0.7 can be observed for some pair of stocks belonging to the same economic sector.

The process of clustering a set of economic entities can improve economic forecasting and modeling of composed financial entities such as, for example, stock portfolios. The most common method of forming homogeneous groups is the principal components analysis of the correlation matrix of the raw data [4]. Correlation based clustering of synchronous financial data sampled at a fixed time horizon has also been performed to obtain a taxonomy of financial data. This has been done by using as a similarity measure the correlation coefficient between two financial time series [5].

Recently [6], it has been proposed to detect the economic information present in a correlation coefficient matrix with a filtering procedure based on the estimation of the subdominant ultrametric [7] associated with a metric distance obtained starting from the correlation coefficient matrix of a set of $n$ stocks. A metric distance can be obtained starting from the correlation coefficient as indicated in Ref. [8]. Having obtained a metric distance one can obtain the minimum spanning tree (MST) and a hierarchical tree associated to each correlation coefficient matrix by using the nearest neighbor single linkage cluster analysis [9]. In other words, geometrical (throughout the MST) and taxonomic (throughout the hierarchical tree) aspects of the correlation present between the stock pairs of a set of stocks can be sorted out using the information contained in the correlation coefficient matrix.
To the best of our knowledge this correlation based clustering method was used for the first time to investigate financial data in Ref. [6].

It is known since 1979 that the degree of intrasector cross-correlation diminishes by diminishing the time horizon used to compute stock returns [10]. This phenomenon is sometime addressed as "Epps effect". The existence of this phenomenon motivates us to investigate the nature and the properties of the hierarchical organization obtained starting from the correlation coefficient matrix as a function of the time horizon used to obtain it.

In the present study, we use the high-frequency data of the transactions occurring in the US equity markets which are recorded in the Trade and Quote database of the New York Stock Exchange. By using this database we are able to investigate comovements of a set of highly capitalized stocks for daily and intra daily time horizons.

The decrease of the cross-correlation between the stock returns observed for diminishing time horizons progressively changes the nature of the hierarchical structure associated to each different time horizon. The structure of the considered set of stocks changes moving from a complex organization to a progressively elementary one when the time horizon of price changes varies from $d=6$ $\mathrm{h}$ and $30 \mathrm{~min}$ to $d / 20=19 \mathrm{~min}$ and $30 \mathrm{sec}$, where $d$ is the daily time horizon at the New York Stock Exchange.

\section{HIERARCHICAL STRUCTURE IN A PORTFOLIO OF STOCKS}

Correlation matrices of price changes have been recently investigated by physicists within the framework of random matrix theory 11, 12]. Here we use the method proposed in Ref. [6] to filter the economic information stored in the correlation coefficient matrix in a way which is straightforward and independent of any exter- 
nal threshold. Specifically, the filtering is done (i) by starting from the synchronous correlation coefficient of the difference of logarithm of stock price computed at a selected time horizon, (ii) by calculating a metric distance between all the pair of stocks and (iii) by selecting the subdominant ultrametric distance associated to the considered metric distance.

The correlation coefficient is defined as

$$
\rho_{i j}(\Delta t) \equiv \frac{<Y_{i} Y_{j}>-<Y_{i}><Y_{j}>}{\sqrt{\left(<Y_{i}^{2}>-<Y_{i}>^{2}\right)\left(<Y_{j}^{2}>-<Y_{j}>^{2}\right)}}
$$

where $i$ and $j$ are numerical labels of the stocks, $Y_{i}=$ $\ln S_{i}(t)-\ln S_{i}(t-\Delta t), S_{i}(t)$ is the value of the stock price $i$ at the trading time $t$ and $\Delta t$ is the time horizon. For each value of $\Delta t$, the correlation coefficient for logarithm price differences (which almost coincides with stock returns) is computed between all the possible pairs of stocks present in the considered portfolio. The empirical statistical average, indicated in this paper with the symbol $<$. $>$, is here a temporal average always performed over the investigated time period.

By definition, $\rho_{i j}(\Delta t)$ can vary from -1 (completely anti-correlated pair of stocks) to 1 (completely correlated pair of stocks). When $\rho_{i j}(\Delta t)=0$ the two stocks are uncorrelated. The matrix of correlation coefficient is a symmetric matrix with $\rho_{i i}(\Delta t)=1$ in the main diagonal. Hence for each value of $\Delta t, n(n-1) / 2=4950$ correlation coefficients characterize each correlation coefficient matrix completely.

A metric distance between pair of stocks can be rigorously determined [8] by defining

$$
d_{i, j}(\Delta t)=\sqrt{2\left(1-\rho_{i j}(\Delta t)\right)} .
$$

With this choice $d_{i, j}(\Delta t)$ fulfills the three axioms of a metric - (i) $d_{i, j}(\Delta t)=0$ if and only if $i=j$; (ii) $d_{i, j}(\Delta t)=d_{j, i}(\Delta t)$ and (iii) $d_{i, j}(\Delta t) \leq d_{i, k}(\Delta t)+$ $d_{k, j}(\Delta t)$. The distance matrix $\mathbf{D}(\Delta t)$ is then used to determine the MST connecting the $n$ stocks.

The MST, a theoretical concept of graph theory [13], is a graph with $n-1$ links which selects the most relevant connections of each element of the set. The MST allows to obtain, in a direct and essentially unique way, the subdominant ultrametric distance matrix $\mathbf{D}^{<}(\Delta t)$ and the hierarchical organization of the elements (stocks in our case) of the investigated data set.

The subdominant ultrametric distance between $i$ and $j$ objects, i.e. the element $d_{i, j}^{<}$of the $\mathbf{D}^{<}(\Delta t)$ matrix, is the maximum value of the metric distance $d_{k, l}$ detected by moving in single steps from $i$ to $j$ through the path connecting $i$ and $j$ in the MST. The method of constructing a MST linking a set of $n$ objects is direct and it is known in multivariate analysis as the nearest neighbor single linkage cluster analysis [9]. A pedagogical exposition of the determination of the MST in the contest of financial time series is provided in Ref. [15].
Subdominant ultrametric space [7] has been fruitfully used in the description of frustrated complex systems. The archetype of this kind of systems is a spin glass [14]. In Ref. [6] the 'ansatz' that the subdominant ultrametric space may reveal part of the economic information stored in the time series of the investigated set was proposed.

\section{EMPIRICAL RESULTS}

We investigate a set of 100 highly capitalized stocks traded in the major US equity markets during the period January 1995 - December 1998. At that time, most of them were used to compute the Standard and Poor's 100 index. The prices are transaction prices stored in the Trade and Quote database of the New York Stock Exchange. The amount of information processed consists of about 100 millions of transactions.

The time horizons investigated in the present study are $\Delta t=d=6 \mathrm{~h}$ and $30 \mathrm{~min}$ (a trading day time interval), $\Delta t=d / 2=3 \mathrm{~h}$ and $15 \mathrm{~min}, \Delta t=d / 5=1 \mathrm{~h}$ and 18 $\min , \Delta t=d / 10=39 \mathrm{~min}$ and $\Delta t=d / 20=19 \mathrm{~min}$ and $30 \mathrm{sec}$. With this choice, in addition to the daily time horizon, we also investigate intra-day time horizons which are progressively shorter.

The daily mean number of transactions for the 100 selected stocks is ranging from 11944.3 transactions of Intel Corp. (INTC) to the 121.48 transactions of Mallinckrodt Inc. New (MKG). In the following we will use the tick symbols to label the various stocks composing our set. Information about the company indicated by each tick symbol can be easily find in several financial web pages such as, for example, http://www.quicken.com. To assess the typical amount of the spreading around the mean value observed for the daily number of transactions we give the minimum and the maximum daily number of transactions occurring for the two companies considered above during the investigated period. Specifically the minimum number of daily transactions was 1717 for INTC and 22 for MKG whereas the maximum was 95437 for INTC and 388 for MKG. By considering that the minimum number of daily transactions is above 17 for the 100 stocks considered in the present study, we decide to investigate time horizons as short as $d / 20$ to statistically ensure that at least 1 transaction occurs during the time horizon $\Delta t$.

In Fig. 1 we show the hierarchical tree obtained for a $\Delta t=6 \mathrm{~h}$ and $30 \mathrm{~min}$ time horizon. In the figure each vertical line indicates a stock. The sector of each stock is coded with a color code provided in the figure caption. Several clusters are clearly identified. The most prominent are (i) the cluster of energy stocks (the blue cluster), (ii) the cluster of financial stocks (green), (iii) technology cluster (red), (iv) basic materials cluster (violet) and (v) utilities cluster (magenta). Hence a collection of oneday time horizon time series of returns carries information about the economic sector of the stocks considered. Additional information about the form and structure of 
the subdominant ultrametric associated with the metric distance is present in the MST. In Fig. 2 we show the MST obtained for the same time horizon as in Fig. 1. In the MST some stocks, such as for example, BAC, INTC or AEP are linked with several stocks belonging to the same sector (financial, technology and utility sector respectively in the discussed example) whereas others (most notable case is General Electric Co., GE) are linked to stocks of different sectors. In this second case the stock with several links acts as a 'reference' stock at different hierarchically structured distances.

The 'Epps effect' predicts that the intra-sector pair correlation decreases by decreasing the time horizon $\Delta t$. In Fig. 3 we show the mean correlation coefficient $\langle\rho\rangle$ obtained by averaging over the $n(n-1) / 2$ off-diagonal elements of the correlation coefficient matrix. In the same figure, we also show the minimum $\rho_{\min }$ and the maximum $\rho_{\max }$ value of the correlation coefficient observed in the correlation matrix as a function of the time horizon $\Delta t$.

As expected the values of $\rho_{\max },\left|\rho_{\min }\right|$ and $\langle\rho\rangle$ are decreasing when $\Delta t$ decreases. The most prominent correlation weakening is observed for the most correlated pair of stocks (the ones having a correlation coefficient closes to the maximum value $\rho_{\max }$ ). In fact $\rho_{\max }$ decreases from 0.76 to 0.52 when $\Delta t$ changes from $6 \mathrm{~h}$ and $30 \mathrm{~min}$ to $19 \mathrm{~min}$ and $30 \mathrm{~s}$.

The decrease of the correlation between pairs of stocks affects the nature of the hierarchical organization of stocks. The clusters observed in figures 1 and 2 progressively disappear and the arrangement of the hierarchical and minimum spanning tree moves from a structured and clustered set to a simpler set. Figures 4 and 5 show the hierarchical tree and the minimum spanning tree observed when the time horizon is set to the minimal value investigated $(\Delta t=19 \mathrm{~min}$ and $30 \mathrm{~s})$. The number of clusters is diminished and also the number of stocks belonging to the remaining ones has decreased. The biggest clusters that are recognizable in figures 4 and 5 are the technology cluster (red lines in Fig. 4 and stocks at the left side of Fig. 5), the financial cluster (green lines in Fig. 4 and stocks on the right of Fig. 5) and two energy clusters (blue lines in Fig. 4 and stocks at the bottom and on the right top of Fig. 5). The change of structure of the MST is indeed dramatic if one considers the role of some 'reference' stock such as, in the present case, GE. It acts as a hierarchical reference for 17 stocks (17 links in the MST) when $\Delta t=6 \mathrm{~h}$ and 30 min whereas it acts as a reference for 61 stocks when the time horizon is decreased to $\Delta t=19 \mathrm{~min}$ and $30 \mathrm{~s}$.

It is worth pointing out that the change in the structure of the MST and hierarchical tree is not just a simple consequence of the 'Epps effect'. In fact the changes observed in the structure of the MST and of the hierarchical tree suggest that the intrasector correlation decreases faster than intersector correlation between pairs of stocks of the considered portfolio. To verify this hypothesis we considered the six largest groups of stocks belonging to the same industrial sector and, for each time horizon, we measured both the intrasector mean value $\left(<\rho^{(i n)}>\right)$ and the intersector mean value $\left(<\rho^{(o u t)}>\right)$ of the correlation coefficient. For a set of $n_{s}$ stocks belonging to a specific sector the average of $\rho^{(i n)}$ is done by considering $n_{s}\left(n_{s}-1\right) / 2$ correlation coefficient whereas the average of $\rho^{(\text {out })}$ involves $\left(n-n_{s}\right) n_{s}$ elements of the correlation coefficient matrix. In Fig. 6 we show the values of $\left\langle\rho^{(\text {in })}>-<\rho^{(\text {out })}>\right.$ as a function of $\Delta t$ for the technology $\left(n_{s}=17\right)$, services $\left(n_{s}=13\right)$, basic materials $\left(n_{s}=11\right)$, financial $\left(n_{s}=10\right)$, consumer non-cyclical $\left(n_{s}=10\right)$ and energy $\left(n_{s}=8\right)$ sector. In all the considered sectors it is evident that the decrease of the degree of correlation is faster at an intrasector level rather than at an intersector level. The different modification of the degree of correlation at intersector or intrasector level confirms that a hierarchical structuring of a stock portfolio occurs when $\Delta t$ increases.

The change of structure of the hierarchical and minimum spanning tree occurs gradually with the decrease of the time horizon. To support this statement, in Table I we report the number of links of a group of stocks as a function of the time horizon. The listed stocks are chosen by selecting the stocks having more than one link when $\Delta t=6 \mathrm{~h}$ and $30 \mathrm{~min}$. The Table shows that several stocks diminish the number of their links in the minimum spanning tree when the time horizon decreases from $\Delta t=d$ to $\Delta t=d / 20$. Exceptions to this general trend are expected because the total number of links of a MST $(n-1)$ is a conserved quantity. The key exception is GE (General Electric Co). The number of links of GE increases gradually from 17 to 61 . In other words, the organization of the MST in several clusters observed in Fig. 2 is gradually lost when the time horizon is decreased. In parallel to the disruption of local clusters a star-like organization with GE at the center of the star progressively emerges (see Fig. 5).

To assess the statistical robustness of our results we perform several tests devoted to - (i) check the significance of the results obtained by comparing them with the ones obtainable starting from surrogate data, (ii) estimate the role of the outliers in the correlation based clustering of the considered portfolio and (iii) consider the stability of the results across time.

In Fig. 7 we show the mean length (top panel) and its standard deviation (bottom panel) of the link of the minimum spanning tree as a function of the time horizon $\Delta t$. We investigate real data and surrogate Gaussian data having the same correlation coefficient matrix as in the real case for the shortest time horizon. Real data are shown as black circles whereas empty circles indicates the results obtained with surrogate data. The error bars shown in the case of surrogate data are one standard deviation in the considered variable. This value is obtained by repeating the generation of surrogate data 100 times. The investigation of surrogate data clearly shows that the decrease of the distance of stock pairs as a function of the time horizon is a real effect that cannot be mimicked 
by surrogate data. The same conclusion is reached when the standard deviation of the link length of the minimum spanning tree is considered. The bottom panel of Fig. 2 shows that the increase of the standard deviation of $d_{i, j}^{<}$ observed in real data, which is a simple measure of the increasing complexity of the MST and hierarchical tree, cannot be reproduced by surrogate data.

The estimation of the correlation coefficient is rather sensitive to outliers. To check how our results are affected by them we repeat our investigation by considering only returns which are in absolute value less than $k \sigma_{\Delta t}$ where $\mathrm{k}$ is set to be $1,2,3,5$ and 10 and $\sigma_{\Delta t}$ is the standard deviation of all returns of the 100 stocks observed for the selected time horizon. In our test the returns which are, in absolute value, larger than the chosen cutoff are set to zero in the calculation of the correlation coefficient. We verify that 65 of 99 links are still observed when we consider the rather severe cutoff induced by setting $\mathrm{k}=3$ and $\Delta t=19 \mathrm{~min}$ and $30 \mathrm{~s}$. For the same parameters but for a 1 trading day time horizon the number of conserved links is 72 . When the cutoff is progressively increased the number of conserved links increases up to 90 (for $\Delta t=19$ min and $30 \mathrm{~s}$ ) and 87 (for $\Delta t=6 \mathrm{~h}$ and $30 \mathrm{~min}$ ). In summary the results obtained are not due to the outliers but rather the large majority of links are reflecting the presence of a synchronization down to the scale of few standard deviation of the ensemble return.

The stability of our results across different averaging period is tested by repeating some of the analyses done by dividing the averaging time interval in subintervals. We verify that a stability of statistical nature is present in our results. For example, by investigating the coordination number of GE yearly for $\Delta t=19 \mathrm{~min}$ and $30 \mathrm{~s}$, we observe that this number is 19 in 1995, 40 in 1996, 52 in 1997 and 48 in 1998 and that the stock GE is always the stock with the largest number of links of the MST. Hence its role of 'hierarchical reference' stock is confirmed for each year of the time period of our database.

\section{A CASE-STUDY: THE STOCKS OF ENERGY SECTOR}

The progressive structuring of the hierarchical tree as a function of the time horizon is shown in Fig. 8 for the representative case of energy sector stocks. In this figure we show the hierarchical trees obtained for the 5 investigated time horizons and we indicate with black lines stocks belonging to the energy sector whereas the remaining lines indicating all the other stocks are left gray.

In Fig. 8a ( $\Delta t=19 \mathrm{~min}$ and $30 \mathrm{~s})$ seven of the eight stocks belonging to the energy sector are observed in two distinct clusters. The first cluster is composed of $\mathrm{XON}, \mathrm{MOB}, \mathrm{CHV}$ and $\mathrm{ARC}$, whereas the second comprises SLB,HAL and BHI. The last stock (OXY) is not directly connected to a stock of the energy sector (it is connected to the 'reference' stock GE). It may be worth pointing out that the stocks SLB, HAL and BHI are all working in the sub-sector oil services and equipment of the energy sector. In Fig. $8 \mathrm{~b}(\Delta t=39 \mathrm{~min})$ almost the same behavior as in Fig. 8a is observed. The only difference being in the increase of the degree of correlation between stocks (manifested as a decrease of the ultrametric distance in the hierarchical tree). Fig. $8 \mathrm{c}$ show the first important change. At this time horizon $(\Delta t=1$ $\mathrm{h}$ and $18 \mathrm{~min}$ ), seven of the eight stocks of the energy sector form a single cluster which is distinct from the others up to a ultrametric distance of $d^{<}=1.15$. This cluster splits in two sub-clusters composed as (i) SLB, HAL and BHI and (ii) XON, MOB, CHV and ARC. In Fig. $8 \mathrm{~d}$ we observe the complete formation of the energy sector cluster. In fact when $\Delta t=3 \mathrm{~h}$ and $15 \mathrm{~min}$ all the eight energy stocks belonging to the energy sector form a single cluster. The cluster is maintaining the internal structure as discussed before with the last stock (OXY) joining the cluster. In Fig. 8e we show the hierarchical tree obtained by using a 1 day time horizon $(\Delta t=6 \mathrm{~h}$ and $30 \mathrm{~min}$ ). The energy cluster is still there with a new stock linking at a longer distance. This stock, indicated as a gray line just at the right of the energy cluster, is the CGP stock. The CGP stock is classified by Forbes in the utilities sector and its main sub-sector is natural gas utilities.

This case study shows that the nature, size and interrelation between the stocks of a given economic sector are affected by the value of the time horizon used to compute the stock return. Specifically time is needed before the cluster organization between stocks of the same sectors takes place completely. Our investigation suggests that an intra-day dynamics is present when the process of the rational price formation of a given financial asset is going on.

\section{DISCUSSION}

Our investigations of the hierarchical structure observed in a set of stocks frequently traded in the main US equity markets show that the degree and nature of crosscorrelation between pairs of stock returns varies as a function of the time horizon used to compute them. Specifically intra-day time series of returns show a lowest degree of correlation that manifest itself in the partial disruption of the hierarchical structure observed for longer (e.g. daily) time horizons. The disruption of the correlation structure is more pronounced for intrasector correlation than for intersector correlation inducing a modification in the correlation based clustering of a stock portfolio whose results become time horizon dependent.

In fact, at shorter time horizons a few stocks (in the present case a single stock) become a hierarchical reference of the large majority of stock. The stock that takes this role is the stock of General Electric Co. in the in- 
vestigated set and time period. The determination of a hierarchical structure for each time horizon allows to follows the mechanism of cluster formation as a function of the time horizon. In the investigated set, the minimum time horizon needed to observe the complete clustering of the eight energy stocks is $3 \mathrm{~h}$ and $15 \mathrm{~min}$ (half trading day). However, this does not implies that until that time horizon correlation between returns of energy stocks are negligible. Indeed, sub-clusters composing the overall energy cluster are observed for time horizon as short as 19 min and $30 \mathrm{~s}$. We interpret these fine details of the cluster formation as an evidence that the process of rational price formation takes a finite amount of time to occur.

We conclude by discussing the reason for selecting a similarity measure which obeys the axioms of a metric distance. By selecting as a similarity measure a metric distance one ensures that the ultrametric structure of the MST is the subdominant ultrametric structure. By translating the mathematical language in common language, the detected subdominant ultrametric is the ultrametric structure which is closer to the original metric structure. This implies that the filtering of information performed by moving from the metric distance matrix of $n(n-1) / 2$ distinct elements to the subdominant ultrametric distance matrix characterized by $n-1$ elements is the only filtering procedure based on the single linkage cluster analysis that allows the minimum lost of information. In other words, under the constraint of reducing the number of elements of the matrix of interest from $n(n-1) / 2$ to $n-1$, the use of by a similarity measure which is a distance ensures that the correlation based clustering retains after filtering the maximal amount of information compatible with the procedure.

\section{ACKNOWLEDGEMENTS}

The authors thank INFM and MURST for financial support. This work is part of the FRA-INFM project 'Volatility in financial markets'. G. Bonanno and F. Lillo acknowledge FSE-INFM for their fellowships. The authors wish to thank Dr. Roberto Renó for a discussion about the "Epps effect".

[1] H. Markowitz, Portfolio Selection: Efficient Diversification of Investment (J. Wiley, New York, 1959).

[2] E. J. Elton and M. J. Gruber, Modern Portfolio Theory and Investment Analysis (J. Wiley and Sons, New York, 1995).

[3] J. Y. Campbell, A. W. Lo, A. C. MacKinlay The Econometrics of Financial Markets, (Princeton University Press, Princeton, 1997).
[4] E. J. Elton and M. J. Gruber, Journal of Business 44, 432-450 (1971).

[5] D. B. Panton, V. Parker Lessig, and O. M. Joy, Journal of Financial and Quantitative Analysis 11, 415-432 (1976).

[6] R. N. Mantegna, Eur. Phys. J. B 11, 193-197 (1999).

[7] R. Rammal, G. Toulouse, and M. A. Virasoro, Rev. Mod. Phys. 58, 765-788 (1986).

[8] J. C. Gower, Biometrika 53, 325-338 (1966).

[9] K. V. Mardia, J. T. Kent, and J. M. Bibby Multivariate Analysis, (Academic Press, San Diego, CA, 1979).

[10] T.W. Epps, Journal of American Statistical Association 74, 291-298 (1979).

[11] L. Laloux, P. Cizeau, J.-P. Bouchaud, and M. Potters, Phys. Rev. Lett. 83, 1467-1470 (1999).

[12] V. Plerou, P. Gopikrishnan, B. Rosenow, L. A. N. Amaral, and H. E. Stanley, Phys. Rev. Lett. 83, 1471-1474 (1999).

[13] D. B. West, Introduction to Graph Theory (Prentice-Hall, Englewood Cliffs NJ, 1996).

[14] M. Mézard, G. Parisi, and M. A. Virasoro, Spin Glass Theory and Beyond (World Scientific, Singapore, 1987).

[15] R. N. Mantegna and H. E. Stanley, An Introduction to Econophysics: Correlations and Complexity in Finance, (Cambridge University Press, Cambridge, 2000).

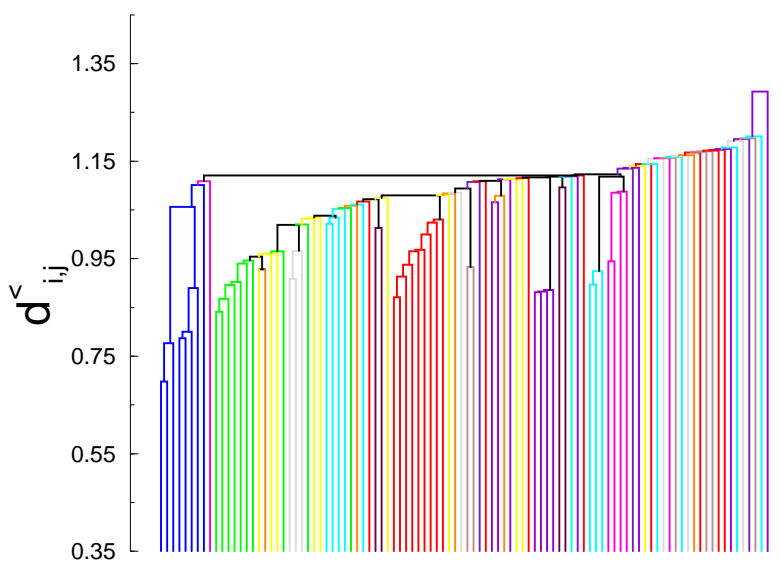

FIG. 1. Hierarchical tree of the set of 100 stocks traded in the US equity markets obtained starting from the return time series computed with a $\Delta t=6 \mathrm{~h}$ and $30 \mathrm{~min}$ time horizon (1 trading day) during the time period Jan 1995-Dec 1998. Each stock is indicated by a vertical line and the color of the line refers to its economic sector as defined by Forbes company. The presence of several clusters of stocks belonging to the same economic sector is clearly seen. Two stocks (lines) links when a horizontal line is drawn between two vertical lines. The height of the horizontal line indicates the ultrametric distance at which the two stocks are connected. The code of colors is the following: energy (blue), utilities (magenta), financial (green), consumer/cyclical (brown), consumer/non-cyclical (yellow), conglomerates (orange), healthcare (gray), services (cyan), technology (red), capital goods (indigo), basic materials (violet) and transportation (maroon). 


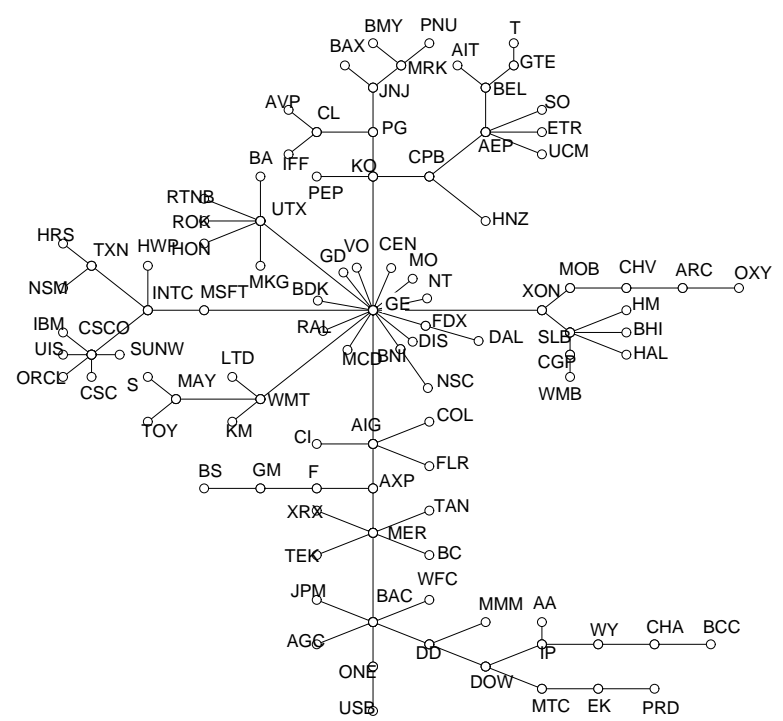

FIG. 2. Minimum spanning tree of the same set of stocks and time horizon used in Fig. 1. Each circle represents a stock labeled by its tick symbol. The minimum spanning tree presents a large amount of stocks having a single link and some stocks having several links. Some of these stocks act as a "center" of a local cluster. Examples are INTC and CSCO for technology stocks, AIG, BAC and MER for financial stocks and AEP for utilities stocks. The stock GE (General Electric Co.) links a relatively large number of stocks belonging to various sectors.

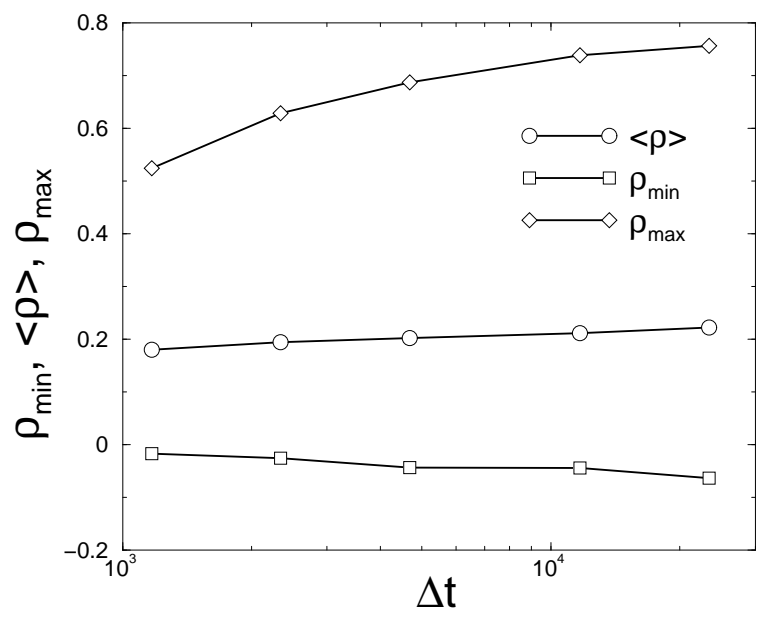

FIG. 3. Mean correlation coefficient $\langle\rho\rangle$, minimum and maximum value of $\rho$ as a function of $\Delta t$. The values are obtained by averaging or searching over the $n(n-1) / 2=4950$ off-diagonal elements of the correlation coefficient matrix computed for the selected time horizon $\Delta t$. The degree of correlation or anti-correlation decreases by decreasing the time horizon. The most prominent decrease is observed for the highest levels of correlation $\left(\rho_{\max }\right)$.

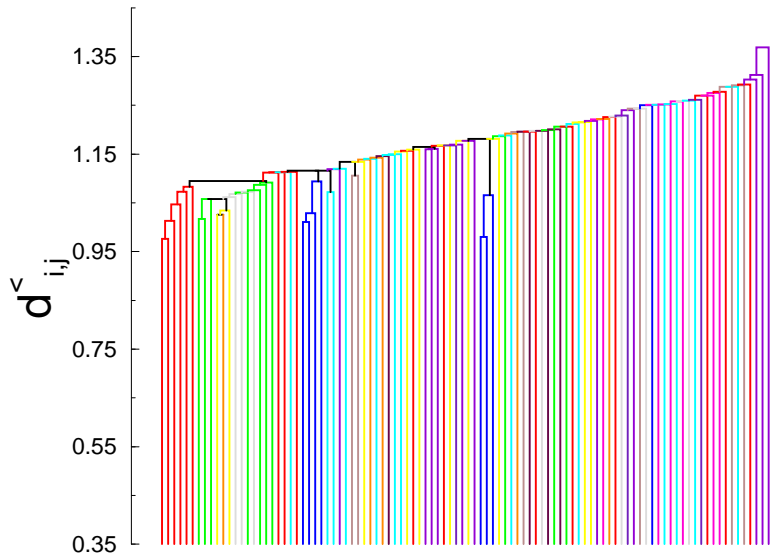

FIG. 4. Hierarchical tree of the set of 100 stocks traded in the US equity markets obtained starting from the return time series computed with a $\Delta t=19 \mathrm{~min}$ and $30 \mathrm{~s}$ time horizon during the time period Jan 1995-Dec 1998. Each stock is indicated by a vertical line and the color of the line refers to its economic sector as defined by Forbes company. The presence of distinct clusters of stocks belonging to the same economic sector is much less evident that in Fig. 1. The code color is the same used for Fig. 1. The biggest clusters are the technology cluster (red lines at the left side of the tree), the financial cluster (green lines) and two energy clusters (blue lines).

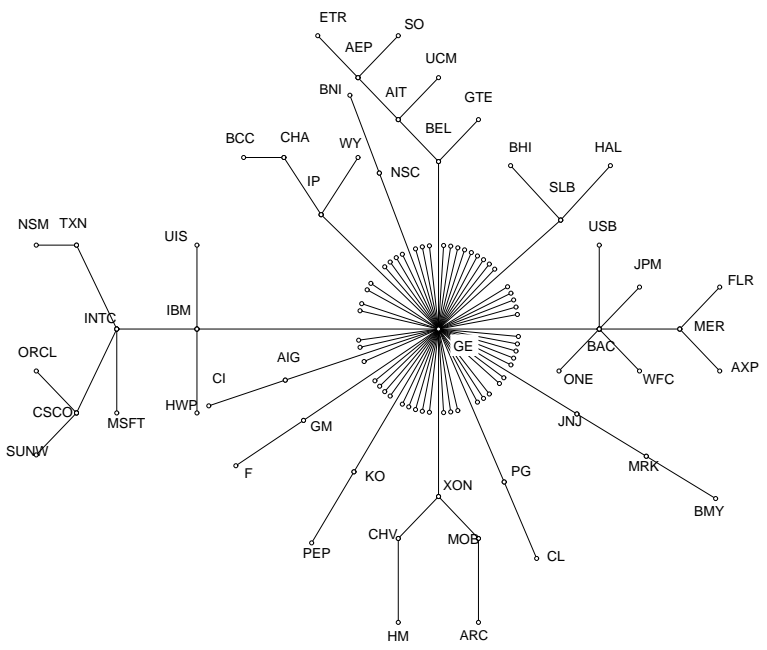


FIG. 5. Minimum spanning tree of the same set of stocks and time horizon used in Fig. 4. Each circle represents a stock labeled by its tick symbol. The minimum spanning tree presents a few cluster composed by companies working in the same sector and by a large amount of stocks having a single link. Most of these stocks connect with a reference stock that, in the present set, is GE. The tick symbols of some stocks composing sector clusters are shown in the figure. The technology cluster is seen at the left side of the graph, the financial cluster on the right side and two energy clusters are identified at the bottom and on the right-top of the graph. The tick symbols of the stocks directly connected with GE are: AA, AGC, AVP, BA, BAX, BC, BDK, BS, CEN, CGP, COL, CPB, CSC, DAL, DD, DIS, DOW, EK, FDX, GD, HNZ, HON, HRS, IFF, KM, LTD, MAY, MCD, MKG, MMM, MO, MTC, NT, OXY, PNU, PRD, RAL, ROK, RTNB, S, T, TAN, TEK, TOY, UTX, VO, WMB, WMT, XRX.

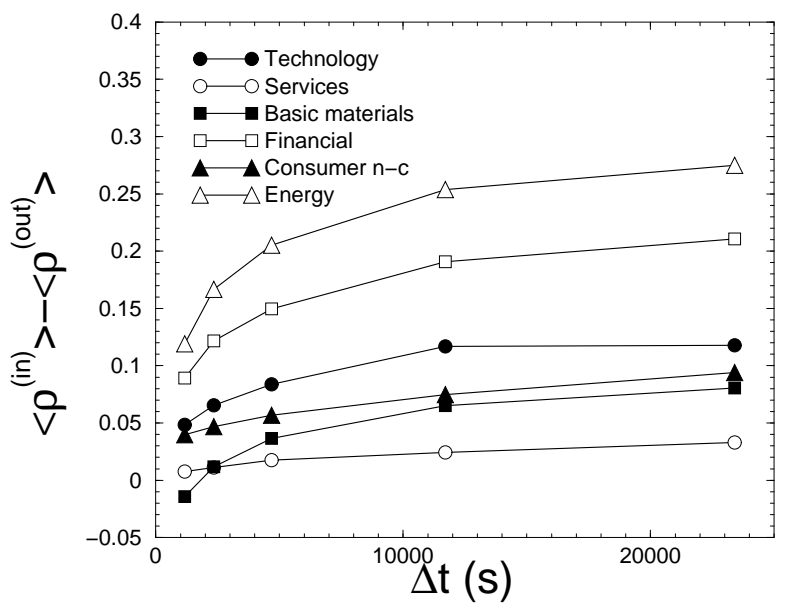

FIG. 6. Intrasector mean correlation coefficient $\left\langle\rho^{(i n)}>\right.$ minus the intersector mean correlation coefficient $\left\langle\rho^{(\text {out })}>\right.$ as a function of the time horizon for the largest groups of stocks present in our investigated set. The "Epps effect" is more pronounced within each sector than outside it. Groups are chosen by using the classification of Forbes. The legend of used symbols is shown in the figure. For the number of stocks belonging to each group see text.

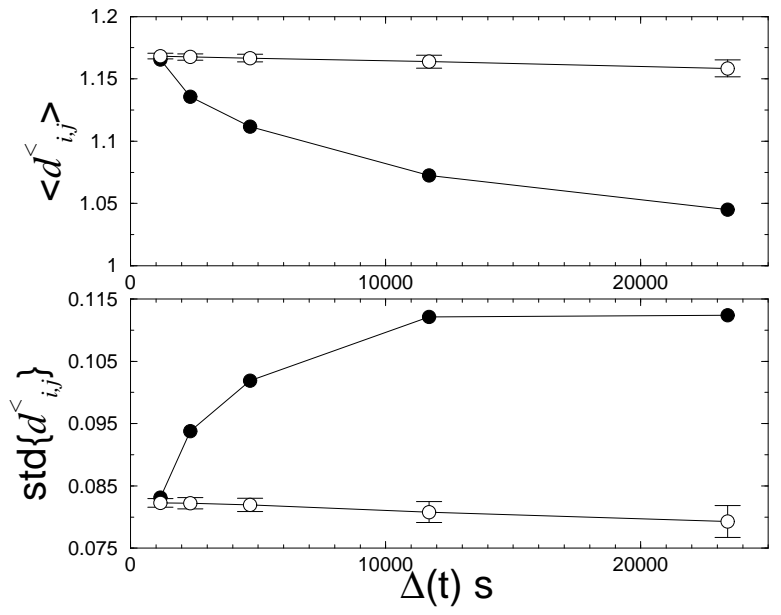

FIG. 7. Mean value (top panel) of the ultrametric distance between each pair of stocks connected in the MST and standard deviation (bottom panel) of the same quantity for real data (black circles) and surrogate data (open circles) generated starting from the correlation coefficient matrix computed for $\Delta t=19 \mathrm{~min}$ and $30 \mathrm{~s}$. The error bars shown in the case of surrogate data are obtained by determining the standard deviation of the investigated quantity over 100 different realizations. By increasing the time horizon the mean value of $d_{i, j}^{<}$ decreases for real data whereas it remains essentially constant for surrogate data. This implies that the degree of correlation of the detected hierarchical structure increases when $\Delta t$ increases only for real data. By increasing $\Delta t$ the standard deviation of $d_{i, j}^{<}$increases for real data whereas it slightly decreases for surrogate data. This behavior shows that the complexity of the clustering of the detected hierarchical structure increases when $\Delta t$ increases only for real data.

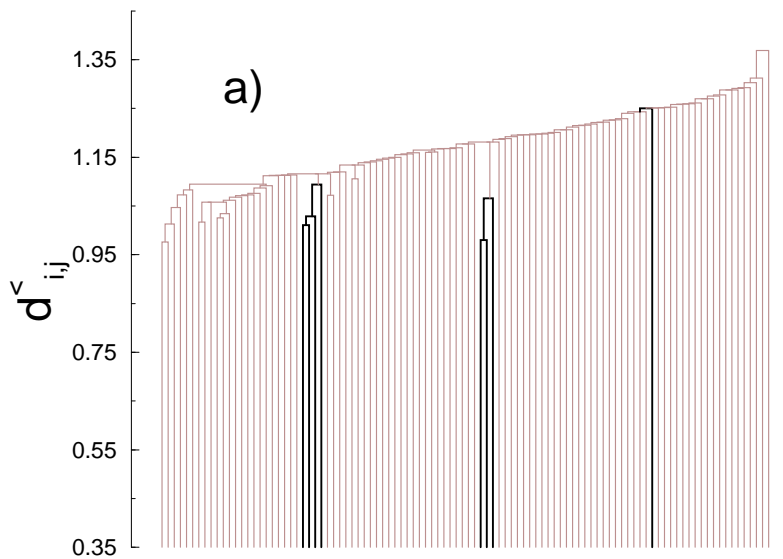



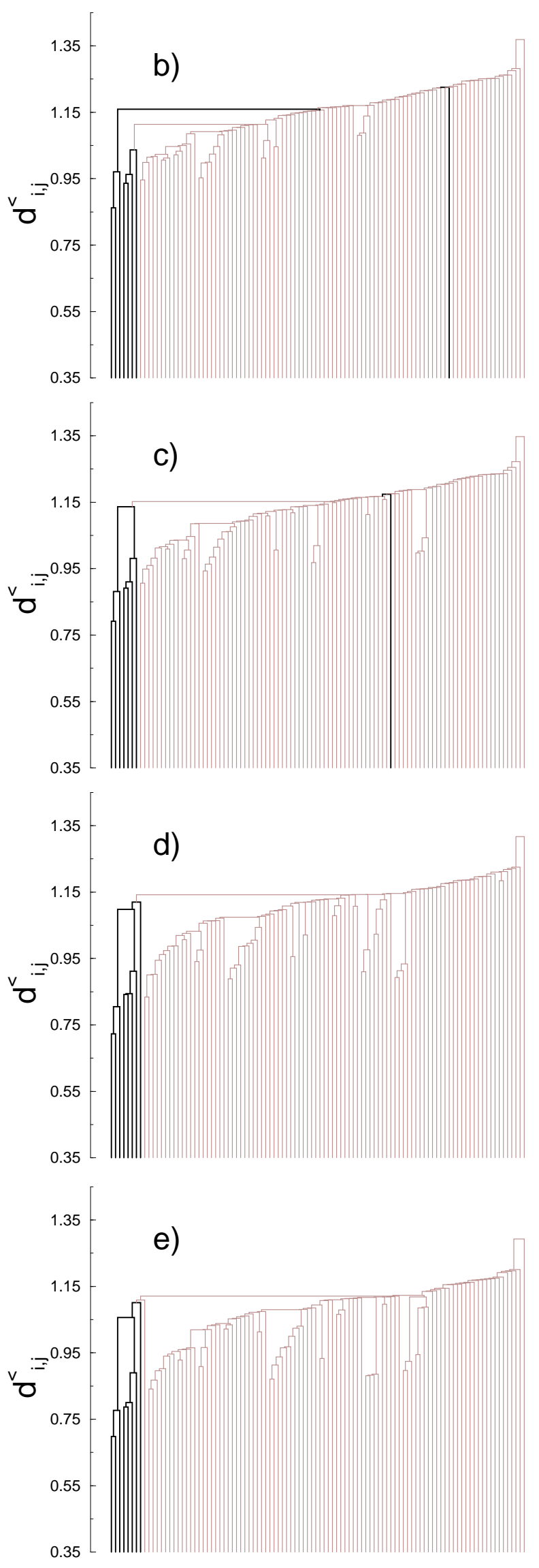

FIG. 8. Progressive structuring of the energy cluster in the hierarchical trees as a function of the time horizon. The hierarchical trees are shown for (a) $\Delta t=d / 20=19 \mathrm{~min}$ and $30 \mathrm{~s}$, (b) $\Delta t=d / 10=39 \mathrm{~min},(\mathrm{c}) \Delta t=d / 5=1 \mathrm{~h}$ and $18 \mathrm{~min}$, (d) $\Delta t=d / 2=3 \mathrm{~h}$ and $15 \mathrm{~min}$ and (e) $\Delta t=d=6 \mathrm{~h}$ and $30 \mathrm{~min}$ (one trading day time interval). Black lines indicate stocks belonging to the energy sector whereas the remaining lines are left gray. In Fig. 6a ( $\Delta t=19 \mathrm{~min}$ and $30 \mathrm{~s})$ two distinct energy clusters are observed. The first cluster is composed of $\mathrm{XON}, \mathrm{MOB}, \mathrm{CHV}$ and $\mathrm{ARC}$, whereas the second comprises SLB,HAL and BHI. The remaining energy stock OXY is disjointed from the two clusters and connected to the 'reference' stock GE. In Fig. 6b ( $\Delta t=39 \mathrm{~min})$ almost the same behavior as in (a) is observed. When $\Delta t=1 \mathrm{~h}$ and $18 \mathrm{~min}$ (Fig. $6 \mathrm{c})$, the two previous clusters merge into a single one which is distinct from all the others up to a ultrametric distance of $d^{<}=1.15$. In Fig.6d the OXY stock joins the energy cluster which maintains its internal structure. In Fig. 6e we finally show the hierarchical tree obtained by using a 1 day time horizon ( $\Delta t=6 \mathrm{~h}$ and $30 \mathrm{~min}$ ). The energy cluster contains ,linked at a longer ultrametric distance, an additional stock (CGP) belonging to the natural gas utilities sub-sector.

TABLE I. Number of links in the minimum spanning tree as a function of the time horizon used to compute return time series. The stocks listed are the stocks having more than one link when $\Delta t=6 \mathrm{~h}$ and $30 \mathrm{~min}$. Several stocks present a number of links that diminish when the time horizon decreases from $\Delta t=6 \mathrm{~h}$ and $30 \mathrm{~min}$ to $\Delta t=19 \mathrm{~min}$ and $30 \mathrm{~s}$. The behavior opposites to this general trend is the one of GE. The number of links of GE increases gradually from 17 to 61 when $\Delta t$ decreases from $6 \mathrm{~h}$ and $30 \mathrm{~min}$ to $19 \mathrm{~min}$ and $30 \mathrm{~s}$.

\begin{tabular}{lccccc}
\hline \hline tick symbol & $\mathrm{d} / 20$ & $\mathrm{~d} / 10$ & $\mathrm{~d} / 5$ & $\mathrm{~d} / 2$ & $\mathrm{~d}$ \\
\hline GE & 61 & 49 & 31 & 20 & 17 \\
BAC & 6 & 7 & 8 & 7 & 6 \\
CSCO & 3 & 3 & 4 & 5 & 6 \\
MER & 3 & 5 & 6 & 6 & 6 \\
UTX & 1 & 1 & 1 & 5 & 6 \\
AEP & 3 & 4 & 4 & 4 & 5 \\
AIG & 2 & 3 & 5 & 5 & 5 \\
SLB & 3 & 3 & 3 & 3 & 5 \\
INTC & 4 & 5 & 5 & 4 & 4 \\
KO & 2 & 2 & 3 & 5 & 4 \\
WMT & 1 & 2 & 3 & 3 & 4 \\
AXP & 1 & 1 & 3 & 3 & 3 \\
BEL & 3 & 4 & 5 & 4 & 3 \\
CL & 1 & 1 & 1 & 3 & 3 \\
CPB & 1 & 1 & 1 & 2 & 3 \\
DD & 1 & 2 & 2 & 3 & 3 \\
DOW & 1 & 1 & 2 & 3 & 3 \\
IP & 3 & 3 & 3 & 3 & 3 \\
JNJ & 2 & 2 & 3 & 3 & 3 \\
May & 1 & 1 & 2 & 3 & 3 \\
MRK & 2 & 2 & 3 & 3 & 3 \\
PG & 2 & 4 & 4 & 2 & 3 \\
TXN & 2 & 2 & 2 & 4 & 3 \\
XON & 3 & 5 & 4 & 3 & 3 \\
ARC & 1 & 1 & 3 & 2 & 2 \\
& & & & &
\end{tabular}




\begin{tabular}{llllll} 
CGP & 1 & 1 & 2 & 2 & 2 \\
CHA & 2 & 2 & 2 & 2 & 2 \\
CHV & 2 & 1 & 1 & 1 & 2 \\
EK & 1 & 1 & 1 & 2 & 2 \\
F & 1 & 2 & 2 & 2 & 2 \\
FDX & 1 & 1 & 1 & 2 & 2 \\
GM & 2 & 1 & 1 & 1 & 2 \\
GTE & 1 & 1 & 1 & 1 & 2 \\
MOB & 2 & 2 & 3 & 5 & 2 \\
MSFT & 1 & 1 & 1 & 3 & 2 \\
MTC & 1 & 1 & 1 & 2 & 2 \\
NSC & 2 & 2 & 2 & 2 & 2 \\
ONE & 1 & 1 & 2 & 1 & 2 \\
WY & 1 & 1 & 1 & 2 & 2 \\
\hline \hline
\end{tabular}

